\title{
Alternative models for genetic evaluation of feed intake by Afrikaner cattle
}

\author{
M.D. MacNeil, ${ }^{1,2,3 *}$ M.M. Scholtz ${ }^{1,2}$, F.J. Jordaan ${ }^{1}$ \& A. Maiwashe ${ }^{1,2}$ \\ ${ }^{1}$ Agricultural Research Council, Private Bag X2, Irene 0062, South Africa \\ ${ }^{2}$ University of the Free State, P.O. Box 339, Bloemfontein 9300, South Africa \\ ${ }^{3}$ Delta G, 145 Ice Cave Road, Miles City, MT 59301, USA
}

(Received 17 May 2019; Accepted 21 August 2019; First published online 17 September 2019)

Copyright resides with the authors in terms of the Creative Commons Attribution 4.0 South African Licence.
See: http://creativecommons.org/licenses/by/4.0/za
Condition of use: The user may copy, distribute, transmit and adapt the work, but must recognise the authors and the South African
Journal of Animal Science.

\begin{abstract}
The objectives of this study were to evaluate statistical models of potential utility for genetic evaluation of feed intake and to estimate the (co)variance components and genetic parameters that would be necessary for their use in a national cattle evaluation scheme. Data were from Afrikaner bulls that were tested between 1974 and 2016 in Phases C ( $n=1250)$ and $D(n=11$ 083) of the National Beef Recording and Improvement Scheme. Statistical analyses in which the number of traits varied and contemporary group effects were considered either random or fixed were conducted using multiple-trait derivative-free restricted maximum likelihood. Contemporary group effects were important. When they were considered random, the estimated phenotypic variance of feed intake increased and its heritability reduced from approximately $0.43 \pm 0.09$ to approximately $0.30 \pm 0.06$. Use of average daily gain, recorded either concurrently with the feed intake measure $\left(A D G_{C}\right)$ or from related bulls reared on-farm $\left(A D G_{D}\right)$, had relatively little effect on estimated heritability of feed intake. Thus, considering contemporary group effects random consistently increased the accuracy of the estimated breeding values. Relatively small genetic correlations between $A D G_{C}$ and $A D G_{D}$ complicated the ultimate application of data recorded in Phase $C$ and the use of data recorded in Phase $D$ in decoupling the phenotypes for feed intake and growth in an evaluation of feed efficiency. Some modification of testing procedures may be necessary to facilitate this application of the results.
\end{abstract}

Keywords: accuracy, contemporary group, genetic parameters, performance testing

\#Corresponding author: macneil.deltag@gmail.com

\section{Introduction}

Converting feedstuffs that are unsuitable for human consumption into animal products is the essence of livestock production. Improving the efficiency of this conversion may result in a reduced carbon footprint and greater sustainability of livestock production (Basarab et al., 2013). Additionally, increased efficiency can increase production, from a finite resource, of highly nutritious food for people.

A variety of metrics have been proposed as measures of efficiency. These include feed conversion ratio, which Armsby (1880) referred to as the 'nutritive ratio', residual feed intake, and residual gain (Koch et al., 1963), residual intake and gain (Berry \& Crowley, 2012). Inherent in all of these measures of efficiency are the component traits feed intake and gain. Yet, combining feed intake and gain to calculate any metric for feed efficiency does not produce additional information relative to that which can be obtained directly from the component traits (Kennedy et al., 1993). Using any of these metrics as a selection criterion fails to promote as much genetic advance in efficiency as an appropriately constructed selection index that incorporates the component traits (Nielsen et al., 2013). Thus, it has been suggested that genetic evaluation schemes that are designed to enhance efficiency of feed utilization should analyse the component traits simultaneously and combine the resulting EBVs to produce an index upon which to base selection decisions (Crews, 2005; Hoque et al., 2006; MacNeil et al., 2011).

Feed intake is most frequently measured in test stations (Bergh, 2010; MacNeil et al., 2011). Owing to the cost of testing, relatively few of the candidates for selection may have a record for feed intake. However, many of their relatives that remain on the farm of origin may have measurements recorded for correlated traits. Combining these records in a multiple-trait genetic evaluation can increase the accuracy of the EBV for feed intake for many more animals than those that have a recorded feed intake measurement. Use of 
multiple traits in the genetic evaluation may also overcome bias due to selection (Pollak et al., 1984) and increase the accuracy of the EBV (Shaeffer, 1984).

Contemporary groups are used to remove biases from genetic evaluations because of differential effects such as weather, which affect performance (VanVleck, 1987). Regarding contemporary groups as fixed removes bias due to association between effects corresponding to the groups and the animals being evaluated. If contemporary groups are considered random, then the effective number of observations is increased at the expense of possible bias. However, this trade-off may be influenced by the particular circumstances, including the number of animals per group and any potential association of genetic merit of the individuals being evaluated with the groups (Ugarte et al., 1992). Schaeffer (2009) contended that contemporary group effects are truly random. Environmental circumstances that condition phenotypic performance cannot be predicted in advance of their occurrence. Still, contemporary group effects are considered fixed in many applications related to many national genetic evaluation schemes (e.g., Su et al., 2017; McHugh et al., 2017).

The goal of this research was to provide Afrikaner breeders with recommendations in order to facilitate their desire to produce more efficient cattle. This goal depends on both collection of appropriate phenotypes and statistical analysis of those phenotypes. Thus, the objectives were i) to estimate variance and covariance components with alternative models that may be used to predict EBV for feed intake that incorporate various combinations of phenotypes and considering contemporary groups as either random of fixed effects; and ii) to assess the accuracy of EBV for feed intake that was estimated using the various models.

\section{Materials \& Methods}

Data used in this research were collected from Afrikaner bulls between 1974 and 2016 in Phases C and D of the National Beef Recording and Improvement Scheme (Bergh, 2010). Briefly, the data collected in Phase $\mathrm{C}$ were from centralized bull testing stations under the supervision of South Africa's Agricultural Research Council. In Phase C, bulls arrived at the testing centre at between 151 and 250 days old. Following a 28-day period for adaptation, they were evaluated over at least 84 days, during which time they were fed ad libitum a growing ration containing $28 \%$ roughage and a maximum of $15 \%$ crude protein and weighed weekly. The data consisted of the individual feed intake (DFI) and average daily gain $\left(A D G_{C}\right)$ of 1 250 bulls. Data collected in Phase D were limited to measures of average daily gain (ADG $)$ from 11083 bulls that were on private farms. At the start of the adaptation period for a Phase $D$ test, the bulls to be evaluated were less than 365 days old and the range in weight within a test group was limited to $65 \mathrm{~kg}$. The length of the adaptation period varied between 21 and 90 days, depending on the anticipated growth rate and source(s) of the bulls. Bulls were weighed bi-weekly and it was stipulated that ADG on-test was $0.4 \mathrm{~kg} / \mathrm{d}$. The bulls tested in Phase $\mathrm{C}$ were mutually exclusive of those tested in Phase D. Contemporary groups (CG) that were composed of one animal were deleted from the dataset. Preliminary analyses of both feed intake and the average daily gains were conducted fitting CG effects, and residuals from each analysis were used to identify potential outliers. Observations with a residual of magnitude greater than 3.0 standard deviations were removed from the dataset. The pedigree of each animal with recorded data was traced back four generations, and this pedigree $(n=27,563)$ was used in the statistical analyses to be described subsequently. Given this pedigree, 80 animals tested in Phase D were unrelated to any animal that had been tested in Phase C.

Six models for genetic evaluation of feed intake by Afrikaner bulls were compared. The single-trait, bivariate, and three-trait models considered contemporary group effects to be either random or fixed. For convenience, these models are referred to as F1, F2, and F $\mathbf{3}$ for the models in which contemporary groups were considered fixed effects, and R1, R2, and R3 for the corresponding models in which contemporary groups were considered random effects. Thus, for example, the three-trait model with random contemporary group effects can be specified explicitly as:

$$
\left[\begin{array}{l}
Y_{1} \\
Y_{2} \\
Y_{3}
\end{array}\right]=\left[\begin{array}{c}
1 \mu_{1} \\
X_{2} \beta_{2} \\
X_{3} \beta_{3}
\end{array}\right]+\left[\begin{array}{l}
Z_{1} u_{1} \\
Z_{1} u_{2} \\
Z_{2} u_{3}
\end{array}\right]+\left[\begin{array}{l}
C_{1} g_{1} \\
C_{1} g_{2} \\
C_{2} g_{3}
\end{array}\right]+\left[\begin{array}{c}
e_{1} \\
e_{2} \\
e_{3}
\end{array}\right],
$$

Where: $Y_{i}=$ a vector of observations for the $i^{\text {th }}$ trait

$i=1$ to 3 for $\mathrm{ADG}_{\mathrm{C}}, \mathrm{DFI}$, and $\mathrm{ADG}_{\mathrm{D}}$, respectively

The $X_{i}, Z_{i}$, and $C_{i}$ are design matrices relating the data to their respective fixed mean and age on test effects, and for $A D G_{D}$ type of test and diet $\left(\beta_{i}\right)$, random additive genetic effects $\left(u_{i}\right)$, and random contemporary group effects $\left(g_{i}\right)$, respectively. Note, for $\mathrm{ADG}_{\mathrm{C}}$ the effect of age on test was found to be 
unimportant $(P=0.54)$ in preliminary analysis and therefore was not included in these models. The $e_{i}$ represent the random residual effects. The random genetic effects were assumed to have null means and variances $(V A R)$ :

$$
V A R\left[\begin{array}{l}
u_{1} \\
u_{2} \\
u_{3}
\end{array}\right]=\left[\begin{array}{ccc}
A \sigma_{u_{1}}^{2} & A \sigma_{u_{1} u_{2}} & A \sigma_{u_{1} u_{3}} \\
A \sigma_{u_{2} u_{1}} & A \sigma_{u_{2}}^{2} & A \sigma_{u_{2} u_{3}} \\
A \sigma_{u_{3} u_{1}} & A \sigma_{u_{3} u_{2}} & A \sigma_{u_{3}}^{2}
\end{array}\right]
$$

Where: $A=$ the numerator relationship matrix with rank equal to the number of animals in the pedigree.

The random contemporary group effects were likewise assumed to have null means and

$$
V A R\left[\begin{array}{l}
g_{1} \\
g_{2} \\
g_{3}
\end{array}\right]=\left[\begin{array}{ccc}
I_{C} \sigma_{g_{1}}^{2} & I_{C} \sigma_{g_{1} g_{2}} & 0 \\
I_{C} \sigma_{g_{2} g_{1}} & I_{C} \sigma_{g_{2}}^{2} & 0 \\
0 & 0 & I_{D} \sigma_{g_{3}}^{2}
\end{array}\right],
$$

Where: $I_{C}$ and $I_{D}=$ identity matrices with rank equal to the number of contemporary groups in Phases $C$ and $\mathrm{D}$, respectively.

Finally, the random residual effects are also assumed to have null means and

$$
V A R\left[\begin{array}{l}
e_{1} \\
e_{2} \\
e_{3}
\end{array}\right]=\left[\begin{array}{ccc}
I_{C} \sigma_{e_{1}}^{2} & I_{C} \sigma_{e_{1} e_{2}} & 0 \\
I_{C} \sigma_{e_{2} e_{1}} & I_{C} \sigma_{e_{2}}^{2} & 0 \\
0 & 0 & I_{D} I \sigma_{e_{3}}^{2}
\end{array}\right],
$$

Where: $I_{C}$ and $I_{D}=$ an identity matrices with rank equal to the number of observations for the traits being analysed from Phases C and D, respectively.

The (co)variance components were estimated using multiple-trait derivative-free restricted maximum likelihood (MTDFREML) (Boldman et al., 1995). This software uses the simplex method (Nelder \& Mead, 1965) to search for parameter estimates that maximize the log of the likelihood function given the data. Convergence was assumed when the variance of the likelihoods in the simplex was less than $10^{-10}$. Herein, accuracy $\left(r_{T 1}\right)$ is defined as the correlation between the true additive genetic value and the EBV. Standard error for the genetic correlations of $A D G_{D}$ with $A D G_{C}$ and $D F I$ were calculated following Bijma \& Bastiaansen (2014) using the average $r_{\mathrm{T}}$ for only those sire families that were represented in both Phase C and Phase D $(n=417)$.

\section{Results and Discussion}

The principles upon which this work is based are well known and long-standing in the science that underpins genetic evaluation of beef cattle. This work was undertaken in recognition that previous genetic evaluations conducted for Afrikaner cattle were based on parameter estimates derived from data collected from Bonsmara (Jordaan, 2015). As points of reference, estimates of $\sigma_{u_{1}}^{2}, \sigma_{u_{1}}^{2}, \sigma_{u_{1} u_{2}}, \sigma_{e_{1}}^{2}, \sigma_{e_{2}}^{2}$, and $\sigma_{e_{1} e_{2}}$ for Bonsmara from a model similar to $\mathrm{F} 2$ were $0.3315,0.0126,0.0429,0.5732,0.0374$, and 0.0807 , respectively (MacNeil et al., 2013). The average ages on test and standard deviations (SD) (in parentheses) for bulls that were evaluated in Phases $C$ and $D$ were 256 days (27 days) and 289 days ( 55 days), respectively. Means and phenotypic standard deviations (SD) of $A D G_{C}$, DFI and $A D_{D}$ were $1.23(0.18) \mathrm{kg} / \mathrm{d}, 8.47(1.13) \mathrm{kg} / \mathrm{d}$, and $0.82(0.21) \mathrm{kg} / \mathrm{d}$, respectively. The intent of this investigation was to assess parameter estimates that could be used in the genetic evaluation of feed intake by Afrikaner (Table 1). These results, together with those from previous studies (e.g., MacNeil et al., 2011; Ceacero et al., 2016; Retallick et al., 2017), document the substantial genetic antagonism between desired growth rate and desired feed intake in improving efficiency. Breaking this genetic antagonism requires measurement of both feed intake and growth. 
Table 1 Estimates of variance components, covariance components, and genetic parameter estimates for six models used in the genetic evaluation of feed intake by young Afrikaner bulls

\begin{tabular}{|c|c|c|c|c|c|c|}
\hline & \multicolumn{6}{|c|}{ Models $^{\mathrm{a}}$} \\
\hline & $\mathrm{F} 1$ & $\mathrm{R} 1$ & $\mathrm{~F} 2$ & $\mathrm{R} 2$ & F3 & R3 \\
\hline \multicolumn{7}{|c|}{ (Co)variance components ${ }^{\mathrm{b}}$} \\
\hline$\sigma_{u_{1}}^{2}$ & 0.3044 & 0.3832 & 0.3081 & 0.4081 & 0.3138 & 0.4231 \\
\hline$\sigma_{u_{2}}^{2}$ & & & 0.0080 & 0.0117 & 0.0080 & 0.0117 \\
\hline$\sigma_{u_{3}}^{2}$ & & & & & 0.0020 & 0.0020 \\
\hline$\sigma_{u_{1} u_{2}}$ & & & 0.0379 & 0.0523 & 0.0392 & 0.0537 \\
\hline$\sigma_{u_{1} u_{3}}$ & & & & & 0.0049 & 0.0072 \\
\hline$\sigma_{u_{2} u_{3}}$ & & & & & 0.0016 & 0.0022 \\
\hline$\sigma_{g_{1}}^{2}$ & & 0.5291 & & 0.5320 & & 0.5322 \\
\hline$\sigma_{g_{2}}^{2}$ & & & & 0.0094 & & 0.0092 \\
\hline$\sigma_{g_{3}}^{2}$ & & & & & & 0.0353 \\
\hline$\sigma_{g_{1} g_{2}}$ & & & & 0.0204 & & 0.0199 \\
\hline$\sigma_{e_{1}}^{2}$ & 0.4116 & 0.3491 & 0.4084 & 0.3290 & 0.4037 & 0.3251 \\
\hline$\sigma_{e_{2}}^{2}$ & & & 0.0140 & 0.0110 & 0.0140 & 0.0110 \\
\hline$\sigma_{e_{3}}^{2}$ & & & & & 0.0081 & 0.0081 \\
\hline$\sigma_{e_{1} e_{2}}$ & & & 0.0409 & 0.0295 & 0.0399 & 0.0285 \\
\hline \multicolumn{7}{|c|}{ Genetic parameters } \\
\hline$h_{1}^{2}$ & $0.43 \pm 0.09$ & $0.30 \pm 0.06$ & $0.43 \pm 0.09$ & $0.32 \pm 0.06$ & $0.44 \pm 0.09$ & $0.33 \pm 0.06$ \\
\hline$h_{2}^{2}$ & & & $0.36 \pm 0.09$ & $0.36 \pm 0.07$ & $0.36 \pm 0.09$ & $0.37 \pm 0.06$ \\
\hline$h_{3}^{2}$ & & & & & $0.20 \pm 0.02$ & $0.04 \pm 0.06$ \\
\hline$r_{1,2}$ & & & $0.76 \pm 0.09$ & $0.76 \pm 0.07$ & $0.78 \pm 0.09$ & $0.77 \pm 0.07$ \\
\hline$r_{1,3}$ & & & & & $0.20 \pm 0.09$ & $0.25 \pm 0.08$ \\
\hline$r_{2,3}$ & & & & & $0.39 \pm 0.09$ & $0.45 \pm 0.08$ \\
\hline
\end{tabular}

\footnotetext{
${ }^{a}$ F1: single trait model for analysis of average daily feed intake (DFI) with contemporary group effects fixed; R1: single trait model for analysis of DFI with contemporary group effects random; F2: bivariate analysis model of average daily gain $\left(A D G_{C}\right)$ and $D F I$ by bulls tested in phase $C$ with contemporary group effects fixed; R2: model similar to $F 2$, but with contemporary group effects random; $F 3$ : three-trait model of $A D G_{C}$ and $D F I$ and average daily gain $\left(A D G_{D}\right)$ by bulls tested in phase D with contemporary groups fixed; and R3: three-trait model of average daily gain and average daily feed intake by bulls tested in phase $C$ and average daily gain by bulls tested in phase $D$ with contemporary groups random

${ }^{\mathrm{b}}$ Subscripts 1,2 and 3 denote DFI, $A \mathrm{DG}_{\mathrm{C}}$, and $\mathrm{ADG}_{\mathrm{D}}$, respectively; $u_{i}=$ indicate additive genetic effects for the $\mathrm{i}^{\text {th }}$ trait; $g_{i}$ $=$ indicate contemporary group effects for the $\mathrm{i}^{\text {th }}$ trait; $e_{i}=$ indicate residual effects for the $\mathrm{i}^{\text {th }}$ trait; $h_{i}^{2}=$ heritability of the $\mathrm{i}^{\text {th }}$ trait; and $r_{i, j}=$ genetic correlation between traits $\mathrm{i}$ and $\mathrm{j}$

Standard errors of estimates follow the \pm symbol
}

In appraising the accuracy of the genetic evaluation for DFI, the results from Phase $C$, in which DFI was measured, and Phase D are addressed separately (Table 2). In Phase C, the average size of CG was 12.3 , but $23 \%$ of the bulls were evaluated in contemporary groups of five or fewer animals. In Phase $D$, the contemporary groups contained between 2 and 76 bulls with an average size of 19 bulls. The most obvious result is that accurate genetic evaluation of DFI requires its measurement, regardless of how the data were analysed, and at present that data recording takes place in Phase C. For bulls tested in Phase C, the average accuracy from the models that treated contemporary groups as being fixed was approximately 0.65 , irrespective of whether the ADG indicator traits were analysed simultaneously. However, in the models where contemporary group effects were considered random, accuracy of the EBV for DFI increased by an average of 0.09 relative to the models in which contemporary groups were considered fixed. Ugarte et al. (1992) concluded that considering CG effects as random when sires are randomly allocated across CG will yield EBV with greater accuracy and a better ranking of animals than fixed models, especially when CG size 
is small. The caveat of sires being randomly allocated across CG is important for the EBV to be unbiased by selection. Herein, the same line of reasoning is extended from sire models to animal models. However, given that the tested bulls were young and did not even have a pedigree estimate of the EBV for feed intake at the time they were nominated for testing, the assumption of randomness seem tenable. The diminishing advantage of treating CG as random in these data is illustrated for models R1 and F1 in Figure 1. Among models, the rank correlations of the EBV for bulls tested in Phase $C$ ranged from 0.934 to 0.999 . Correlations between rankings of EBV from R2 with R3 and F2 with F3 were greatest, followed relatively closely by R1 with R2 and R1 with R3. For bulls tested in Phase $D$, the corresponding advantage from considering contemporary groups to be random was approximately 0.03 . If the trait of interest (DFI) was analysed, the addition of $A D G_{C}$ and $A D G_{D}$ to the analysis as correlated traits was of little benefit to the evaluation of bulls that were tested in either phase. The rank correlation between EBV from R3 and F3 for bulls evaluated in Phase D was 0.952 compared with a rank correlation of 0.936 between the pedigree EBV from R2 and the EBV from R3.

Table 2 Average accuracy of estimated breeding values for feed intake as a function of number of traits analysed and consideration of contemporary groups as either random or fixed effects

\begin{tabular}{lccccccc}
\hline & \multicolumn{9}{c}{ Models $^{1}$} \\
\cline { 2 - 7 } Source of data & F1 & F2 & F3 & R1 & R2 & R3 \\
\hline Phase C test station & 0.643 & 0.648 & 0.654 & 0.716 & 0.741 & 0.745 \\
Phase D on-farm test & 0.267 & 0.268 & 0.287 & 0.295 & 0.302 & 0.327 \\
\hline
\end{tabular}

1 F1: single trait model for analysis of average daily feed intake (DFI) with contemporary group effects fixed; R1: single trait model for analysis of DFI with contemporary group effects random; F2: bivariate analysis model of average daily gain $\left(A D G_{C}\right)$ and DFI by bulls tested in phase $C$ with contemporary group effects fixed; R2: model similar to $F 2$, but with contemporary group effects random; F3: three-trait model of $A D G_{C}$ and DFI and average daily gain (ADGD) by bulls tested in phase D with contemporary groups fixed; and R3: three-trait model of average daily gain and average daily feed intake by bulls tested in phase $C$ and average daily gain by bulls tested in phase $D$ with contemporary groups random

Genetic evaluations for efficiency traits can be based on indexes of EBV from multiple trait analyses (Kennedy et al., 1993; Thallman et al., 2018). Decoupling recording of average daily gain and feed intake would facilitate reduced cost of data collection by shortening the feed intake test period (Nielsen et al., 2013; Manafiazar et al., 2017; Thallman et al., 2018) with the potential downstream effect of more animals being phenotyped for feed intake at a reduced cost per animal (Culbertson et al., 2015). However, the relatively small genetic correlations between $A D G_{C}$ and $A D G_{D}$ observed with models $F 3$ and $R 3$ are troubling. Nominally, these should be independent measures of the same trait, although recorded here from different animals. It has been widely accepted that traits with a correlation $>0.80$ can be assumed to be genetically the same trait (Robertson, 1959). However, neither of the two measures of the genetic correlation of $A D G_{C}$ with $A D G_{D}$ approached this threshold. With additive genetic component of $A D G_{C}$ explaining only approximately $20 \%$ of the genetic variation in $A D G_{D}$, along with the low heritability estimates for $A D G_{D}$, decoupled recording of feed intake from the measurement of average daily gain becomes problematic for indexes of efficiency. Contrary to the present results, Baker et al. (2002) indicated central test station data was useful for ranking bulls on genetic merit for growth in both feedlot and on pasture. Similarly, Johnston et al. (2003) found no evidence of genotype by production environment interactions that affected performance. However, Burrow (2012) cautioned that the absence of interaction between genotype and environment may depend on the magnitude of the environmental effects. A lack of genetic connectedness between animals evaluated in Phases C and D, as has been previously observed for Afrikaner generally (MacNeil et al., 2018), could also potentially give rise to the observed genotype by environment interaction. However, with progeny of 417 sires being evaluated in both phases, a lack of connectedness seems an unlikely explanation for this interaction. With the assumption of fixed contemporary groups (model F3), the efficiency of selection based on $A D G_{C}$ to improve $A D G_{D}$ relative to direct selection on $A D G_{D}$ is predicted to be $58 \%$, if selection intensity is constant. However, with contemporary groups considered random (model R3), indirect selection becomes $129 \%$ as efficient as the selection among bulls tested under the Phase D protocol. Because many more bulls are tested in Phase D than in Phase $C$, the stipulation of equal selection intensity would be unrealistic if a fixed number of bulls are to be selected. In this case, having greater response in $A_{D G_{D}}$ from selection among bulls tested in Phase $\mathrm{C}$ becomes impossible, without significantly increasing the relative number of bulls tested in Phase $\mathrm{C}$. 


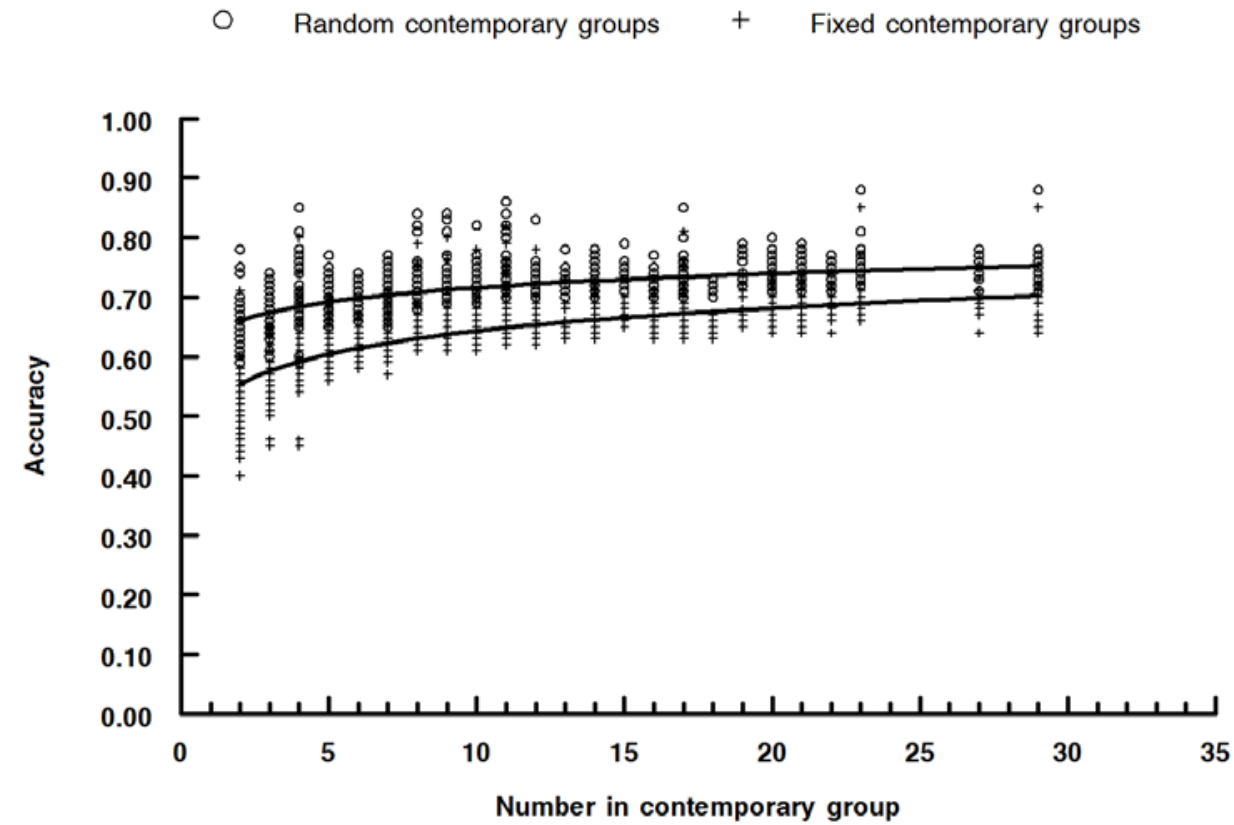

Figure 1 Relationship of accuracy of the estimated breeding values from single-trait models to the number of animals in each contemporary group of the central test stations

\section{Conclusions}

Contemporary group effects were important, so that when they were considered random the estimated phenotypic variance of feed intake was increased and its heritability was reduced. Use of average daily gain, either recorded concurrently with the feed intake measure or from related bulls reared on-farm, had relatively little effect on estimated heritability of feed intake. Thus, considering contemporary group effects to be random consistently increased the accuracy of the EBV. Relatively small genetic correlations between measures of average daily gain complicate the ultimate application of data recorded in Phase $\mathrm{C}$ and the use of data recorded in Phase D in decoupling the phenotypes for feed intake and growth in an evaluation of feed efficiency. Some modification of testing procedures may be necessary in order to increase the usefulness of growth performance as expressed by bulls that are tested in Phase D. Afrikaner breeders would be well advised to coordinate the recording of feed intake data so as to reduce the percentage of bulls that are evaluated in small contemporary groups.

\section{Acknowledgements}

The authors thank the Afrikaner breeders of South Africa for collection of pedigree and phenotype data that were used for this study.

\section{Authors' Contributions}

The study was conceived by MDM and MMS. FJJ oversaw the initial preparation of the data. MDM led the analyses of data and interpretation of results with assistance from MMS and AM. The manuscript was initially drafted by MDM in collaboration with MMS. All authors read and approved the final manuscript.

\section{Conflict of Interest Declaration}

The authors declare that they have no conflicts of interest with regard to this work.

\section{References}

Armsby, H.P., 1880. Manual of cattle-feeding. John Wiley \& Sons, New York, USA.

Baker, J.F., Vann, R.C. \& Neville, Jr., W.E., 2002. Evaluations of genotype $\times$ environment interactions of beef bulls performance-tested in feedlot or pasture. J. Anim. Sci. 80, 1716-1724. Doi: 10.2527/2002.8071716x

Basarab, J.A., Beauchemin, K.A., Baron, V.S., Ominski, K.H., Guan, L.L., Miller, S.P. \& Crowley, J.J., 2013. Reducing GHG emissions through genetic improvement for feed efficiency: effects on economically important traits and enteric methane production. Animal 7, 303-315. Doi: 10.1017/S1751731113000888

Bergh, L., 2010. The national beef recording and improvement scheme. In: M.M. Scholtz (ed). 2nd edition. Beef breeding in South Africa. Agricultural Research Council, Pretoria, South Africa. pp. 55-70. 
Berry, D.P. \& Crowley, J.J., 2012. Residual intake and body weight gain: A new measure of efficiency in growing cattle. J. Anim. Sci. 90, 109-115. Doi: 10.2527/jas.2011-4245

Bijma, P. \& Bastiaansen, J.W., 2014. Standard error of the genetic correlation: How much data do we need to estimate a purebred-crossbred genetic correlation? Genet. Sel. Evol. 46, 79. Doi: 10.1186/s12711-014-0079-z

Boldman, K.G., Kriese, L.A., VanVleck, L.D., Van Tassell, C.P. \& Kachman, S.D., 1995. A manual for use of MTDFREML. A set of programs to obtain estimates of variances and covariances (draft). US Department of Agriculture, Agricultural Research Service, Clay Center, Nebraska.

Burrow, H.M., 2012. Importance of adaptation and genotype $x$ environment interactions in tropical beef breeding systems. Animal 6, 729-740. Doi: 10.1017/S175173111200002X

Ceacero, T.M., Mercadante, M.E.Z., Cyrillo, J.N.S.G., Canesin, R.C., Bonilha, S.F.M. \& de Albuquerque, L.G., 2016. Phenotypic and genetic correlations of feed efficiency traits with growth and carcass traits in Nellore cattle selected for postweaning weight. PLoS ONE 11(8): e0161366. Doi: 10.1371/journal.pone.0161366

Crews Jr., D.H., 2005. Genetics of efficient feed utilization and national cattle evaluation: A review. Genet. Mol. Res. 4, 152-165.

Culbertson, M.M., Speidel, S.E., Peel, R.K., Cockrum, R.R., Thomas, M.G. \& Enns, R.M., 2015. Optimum measurement period for evaluating feed intake traits in beef cattle. J. Anim. Sci. 93, 2482-2487. Doi: 10.2527/jas.2014-8364

Hoque, M.A., Arthur, P.F., Hiramoto, K. \& Oikawa, T., 2006. Genetic relationship between different measures of feed efficiency and its component traits in Japanese Black (Wagyu) bulls. Livest. Sci. 99, 111-118. Doi: 10.1016/j.livprodsci.2005.06.004

Johnston, D.J., Reverter, A., Burrow, H.M., Oddy, V.H. \& Robinson, D.L., 2003. Genetic and phenotypic characterisation of animal, carcass, and meat quality traits from temperate and tropically adapted beef breeds. 1. Animal measures. Aust. J. Agric. Res., 54, 107-118. Doi: 10.1071/AR02085

Jordaan, F.J., 2015. Genetic and environmental trends in landrace beef breeds and the effect on cow productivity. MSc Agriculture dissertation, University of the Free State, Bloemfontein. page 6.

Kennedy, B.W., Van der Werf, J.H.J. \& Meuwissen, T.H.E., 1993. Genetic and statistical properties of residual feed intake. J. Anim. Sci. 71, 3239-3250.

Koch, R.M., Swiger, L.A., Chambers, D. \& Gregory, K.E., 1963. Efficiency of feed use in beef cattle. J. Anim. Sci. 22, 486-494.

MacNeil, M.D., Lewis, R.M. \& Scholtz, M.M., 2018. Degree of connectedness among herds and sires of current candidates for selection in Afrikaner cattle with reported weaning weights. Proc. World Cong. Genet. Appl. Livestk. Prod. Electronic Poster Session - Genetic Gain - Strategies for Local Breeds 2, 34.

MacNeil, M.D., Lopez-Villalobos, N. \& Northcutt, S.L., 2011. A prototype national cattle evaluation for feed intake and efficiency of Angus cattle. J. Anim. Sci. 89, 3917-3923. Doi: 10.2527/jas.2011-4124

MacNeil, M.D., Scholtz, M.M. \& Maiwashe, A., 2013. Estimates of variance components for postweaning feed intake and growth in Bonsmara bulls and evaluation of alternative measures of feed efficiency. S. Afr. J. Anim. Sci. 43, 1824. Doi: $10.4314 /$ sajas.v43i1.2

Manafiazar, G., Basarab, J.A., McKeown, L., Stewart-Smith, J., Baron, V., MacNeil, M.D. \& Plastow, G., 2017. Optimizing feed intake recording and feed efficiency estimation to increase the rate of genetic gain for feed efficiency in beef cattle. Can. J. Anim. Sci. 97, 456-465. Doi: 10.1139/cjas-2016-0118

McHugh, N., Pabiou, T., Wall, E., McDermott, K. \& Berry, D.P., 2017. Impact of alternative definitions of contemporary groups on genetic evaluations of traits recorded at lambing. J. Anim. Sci. 95, 1926-1938. Doi: 10.2527/jas.2016.1344

Nelder, J.A. \& Mead, R., 1965. A simplex method for function minimization. Computer J. 7:308-313.

Nielsen, M.K., MacNeil, M.D., Dekkers, J.C.M. Crews Jr., D.H., Rathje, T.A., Enns, R.M. \& Weaber, R.L., 2013. Lifecycle, total industry genetic improvement of feed efficiency in beef cattle: Blueprint for the Beef Improvement Federation. Prof. Anim. Scient. 29, 559-565. Doi: 10.15232/S1080-7446(15)30285-0

Pollak, E.J., van der Werf, J. \& Quaas, R.L., 1984. Selection bias and multiple trait evaluation. J. Dairy Sci. 67, 15901595.

Retallick, K.J., Bormann, J.M., Weaber, R.L., MacNeil, M.D., Bradford, H.L., ..., Kuehn, L.A., 2017. Genetic variance and covariance and breed differences for feed intake and average daily gain to improve feed efficiency in growing cattle. J. Anim. Sci. 95, 1444-1450. Doi: 10.2527/jas.2016.1260

Robertson, A., 1959. The sampling variance of the genetic correlation coefficient. Biometrics 15, 469-485. Doi: $10.2307 / 2527750$

Schaeffer, L.R., 1984. Sire and cow evaluation under multiple trait models. J. Dairy Sci. 67, 1567-1580.

Schaeffer, $\quad$ L.R., $2009 . \quad$ Contemporary groups are always http://animalbiosciences.uoguelph.ca/ Irs/piksLRS/ranfix.pdf

Su, H., Golden, B., Hyde, L., Sanders, S. \& Garrick, D., 2017. Genetic parameters for carcass and ultrasound traits in Hereford and admixed Simmental beef cattle: Accuracy of evaluating carcass traits. J. Anim. Sci. 95, 4718-4727. Doi: $10.2527 / j a s 2017$

Thallman, R.M., Kuehn, L.A., Snelling, W.M., Retallick, K.J., Bormann, J.M., ..., MacNeil, M.D., 2018. Reducing the period of data collection for intake and gain to improve response to selection for feed efficiency in beef cattle. $\mathrm{J}$. Anim. Sci. 96, 854-866. Doi: 10.1093/jas/skx077

Ugarte, E., Alenda, R. \& Carabano, M.J., 1992. Fixed or random contemporary groups in genetic evaluations. J. Dairy Sci. $75,269-278$.

VanVleck, L.D., 1987. Contemporary groups for genetic evaluations. J. Dairy Sci. 70, 2456-2464. 